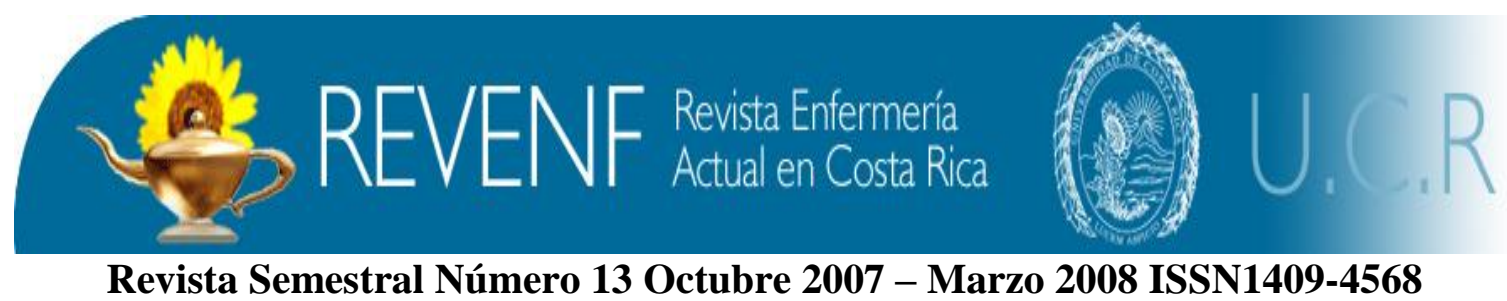

Como citar este artículo

Alfaro Madrigal, Marianne y otros. Sistematización de experiencias de enfermería con personas que padecen trastornos mentales y sus familias. Rev. Enfermería Actual en Costa Rica [en línea].Set 2007/Abril 2008, No.13 [citado (fecha)]. Disponible World Wide Web: < http://www.revenf.ucr.ac.cr/psiquiatria.pdf> ISSN $1409-4568$

\title{
SISTEMATIZACIÓN DE EXPERIENCIAS DE ENFERMERÍA CON PERSONAS QUE PADECEN TRASTORNOS MENTALES Y SUS FAMILIAS $^{1}$
}

\begin{abstract}
RESUMEN
Esta investigación tuvo como propósito sistematizar el desarrollo del proceso de atención de enfermería dirigido a tres personas que han sido diagnosticadas con trastorno mental y sus familias, el cual fue realizado por un grupo de estudiantes y la docente del Módulo de Intervención de Enfermería en la Adultez en Procesos Mentales Mórbidos. La estrategia metodológica para el análisis fue la propuesta de sistematización de Oscar Jara, en la cual se utilizó la técnica del estudio de caso. Se utilizó para recolección de información: revisión del expediente clínico, historial de enfermería, genograma y el apgar familiar, el Cuestionario General de Salud de Golberg (versión corta de 12 items) y se realizó en cada caso el mini examen mental. Las principales situaciones de enfermería encontradas fueron: alteraciones en el patrón del sueño, riesgo de padecer enfermedades crónico-degenerativas, así como estilos de vida inadecuados principalmente relacionados con la nutrición y el ejercicio; prevalencia en los trastornos del afecto y desconocimiento por parte de la persona y su familia sobre los aspectos fundamentales de la enfermedad y el tratamiento. Además, dificultades en la comunicación, baja autoestima, aislamiento social y relaciones familiares poco satisfactorias. Se concluye que es evidente la relación significativa entre los estilos de vida inadecuados y la enfermedad mental, ya que los factores de riesgo tienen mayor prevalencia que los factores protectores, poniendo en desventaja a estas personas, que también presentan otras patologías crónicas degenerativas. Además las relaciones interpersonales poco efectivas pueden ser precursoras y agravantes de la enfermedad mental en las personas, pues presentan disfunciones en la comunicación, en la expresión de afecto y sentimientos hacia los demás, lo que imposibilita la búsqueda de apoyo y una rehabilitación óptima.
\end{abstract}

Marianne Alfaro Madrigal ${ }^{2}$

Valerie Arburola Castillo Amelia Carrillo Medina ${ }^{2}$ Marianela Espinoza Guerrero ${ }^{2}$ Natalia Franco Chacón ${ }^{2}$ Melania Gómez Rojas ${ }^{2}$ Raquel Rojas Posada ${ }^{2}$ Mary Meza Benavides ${ }^{3}$

Palabras clave: enfermería, trastorno psiquiátrico, sistematización, familia, adultez.

\footnotetext{
${ }^{1}$ Fecha de recepción: marzo 2007

Fecha de Aceptación: Julio 2007

2 Alfaro madrigal Marianne, Alburola Castillo Valerie, Carrillo Medina Amelia, Espinoza Guerrero Marianela, Franco Chacón Natalia, Goméz Rojas Melania, Rojas Posada Raquel. Estudiantes de IV año de la carrera de Licenciatura en Enfermería, Universidad de Costa Rica.

${ }^{3}$ Profesora de la Escuela de Enfermería, Universidad de Costa Rica. marymeza1 @ yahoo.com
} 


\begin{abstract}
This investigation had like intention to systematize the development of the process of attention of nursing directed to three people who have been diagnosed with mental disorders and its families, which was made by a group of students and the educational one of the Module of Intervention of nursing in the Adulthood in Mental Illness Processes. The methodological strategy for the analysis was the proposal of systematization of Oscar Jara, in which the technique of the case study was used. It was used for obtaining information: revision of the clinical history file of nursing, genograma and familiar apgar, the General Questionnaire of Health of Golberg (short version of 12 items) and was made in each case the mini mental examination. The main found situations of nursing were: alterations in the pattern of the dream, risk of suffering diseases chronic-degenerative, as well as inadequate styles of life mainly related to the nutrition and the exercise; prevalence in the disorders of the affection and ignorance on the part of the person and her family on the fundamental aspects of the disease and the treatment. In addition, difficulties in the communication, low self-esteem, social isolation and little satisfactory familiar relations. One concludes that the significant relation between the inadequate styles of life and the mental disease is evident, since the risk factors have greater prevalence than the protective factors, putting at a disadvantage these people, who also present other degenerative chronic pathologies. In addition little effective the interpersonal relations can be precursory and aggravating of the mental disease in the people, because they present dysfunctions in the communication, the expression of affection and feelings towards the others, which disables the support search and an optimal rehabilitation.
\end{abstract}

Key words: nursing, psychiatric disorders, systematization, family, adulthood.

\section{Introducción}

La salud mental al igual que el concepto de salud-enfermedad, ha trascendido y evolucionado a través del tiempo; sin embargo, esto ha generado gran controversia debido a los diferentes significados que cada región le ha atribuido, dada su situación cultural y contexto histórico.

De acuerdo con lo anterior la OPS (2004) menciona que la salud mental abarca los aspectos del bienestar subjetivo, la percepción de la propia eficacia, la autonomía, la competencia, la dependencia intergeneracional y la autorrealización de las capacidades intelectuales y emocionales del ser humano; sin embargo, esta concepción es muy relativa, ya que se relaciona directamente con la historia de la persona y las experiencias previas de esta.

Por otra parte, la Organización Mundial de la Salud (2001) aporta estadísticas interesantes para reflexionar la situación actual de la salud mental, ya que dicha organización afirma que el $25 \%$ de la población mundial ha sufrido o sufre de trastornos mentales; de la misma manera estos representan el $12 \%$ de la carga de 
morbilidad misma que para el año 2020 se estima que aumente a un $15 \%$. Cabe destacar que estos registros de salud mental se enfocan únicamente en la morbilidad y la mortalidad de la población, incluyendo para ello exclusivamente a las personas consultantes de los servicios hospitalarios; lo que puede remitir a la incógnita de la cantidad de aquellos (as) individuos que no acuden a un centro de salud para su asistencia.

Ahora bien, aunque estos datos reflejan las condiciones mentales a nivel mundial, Costa Rica no escapa de esta realidad, ya que dentro de lo mencionado por la OPS (2004) se expone lo acontecido en la Caja Costarricense del Seguro Social (CCSS), donde se afirma que las acciones de las autoridades de salud se basan principalmente en el tratamiento de la enfermedad psiquiátrica, la cual es atendida en su mayoría por el tercer nivel. De ahí que, datos obtenidos según una serie de encuestas realizadas en el periodo 1987 2002, a partir de la consulta externa psiquiátrica de la Caja Costarricense Seguro Social revelen que los trastornos mentales más frecuentes son los trastornos neuróticos en primer lugar, las psicosis en segundo, el alcoholismo en tercero y finalmente, la dependencia y el abuso de drogas el cuarto lugar. (OPS, 2004).

Este panorama, intrínsecamente da pie a señalar que los motivos de consulta, pueden ser detectados de una forma temprana desde la atención primaria, sin necesidad de recurrir al tercer nivel.

Esto se fundamenta en las Políticas de Salud las cuales destacan que:

"El fomento de las acciones orientadas a la promoción de la salud mental así como a la

\begin{abstract}
prevención, tratamiento y rehabilitación de los trastornos mentales en los diferentes niveles de atención del sistema nacional de salud deben tener énfasis en el primer nivel y priorizar la salud mental" (Ministerio de Salud 2003: p. 46).
\end{abstract}

Lamentablemente el cumplimiento de estas políticas se ve obstaculizado, porque no se destinan adecuadamente recursos humanos y económicos para que se respeten; además no hay apoyo institucional ni coordinación entre los sectores involucrados en el tema. De la misma manera la inversión destinada a la salud mental está dirigida básicamente a la curación, no así a la promoción del autocuidado y a la prevención de las enfermedades mentales, y a la rehabilitación.

Bajo estas circunstancias se plantea la importancia de la intervención de un equipo interdisciplinario en salud para la atención de salud mental en el primer nivel; $y$ en este caso de manera específica del profesional de enfermería, el o la cual posee las herramientas necesarias para el abordaje integral de la persona con trastorno mental, donde se fortalezca el autocuidado de la salud con el objetivo de disminuir las posibilidades de recaídas, complicaciones o reingresos hospitalarios, así como el fomento de la calidad de vida de las personas.

A partir de lo planteado anteriormente, se realizó una investigación desde la disciplina enfermera, en la cual se buscó crear conocimiento, a partir de la siguiente interrogante:

¿Cuál es la vivencia de las personas en relación con un trastorno mental y cómo se desarrolla la intervención de un grupo de estudiantes de enfermería para el abordaje de dichas personas, en tres familias de un área de salud de la región metropolitana de Costa Rica? 
Ahora bien, se dio respuesta a la interrogante planteada con la guía de los siguientes objetivos:

Objetivo general: Desarrollar el proceso de atención de enfermería dirigido a tres personas que han sido diagnosticadas con trastorno mental y sus familias, con el propósito de realizar una etapa de valoración y a partir de ésta elaborar, implementar y evaluar el plan de atención.

\section{Objetivos específicos:}

1. Realizar una valoración de enfermería sobre la vivencia de la persona y su familia en relación con el trastorno mental.

2. Establecer las principales características sociodemográficas y familiares de las tres personas estudiadas.

3. Identificar las situaciones de enfermería encontradas en cada una de las familias y personas atendidas.

4. Determinar las principales acciones de enfermería que se ejecutaron en las situaciones encontradas.

5. Evaluar los resultados de las acciones implementadas.

6.

La presente investigación correspondió a una investigación cualitativa con metodología de sistematización de experiencias. Para Jara (1994), la sistematización consiste en la interpretación crítica de experiencias, que mediante su organización y reconstrucción evidencia la lógica de lo vivenciado, los elementos que han intervenido en este proceso, la relación que existe entre estos, y el por qué de dicha relación. Este mismo autor afirma que la sistematización de experiencias se guía por cinco pasos o tiempos, a saber: el punto de partida, el establecimiento de preguntas iniciales, la recuperación del proceso vivido, la realización de una reflexión de fondo y finalmente, la formulación de los puntos de llegada.

Las experiencias que se tomaron en cuenta para dicho estudio, fueron obtenidas de la práctica: Procesos Mentales Mórbidos en la Adultez, la cual tiene como propósito que los y las estudiantes de enfermería analicen los determinantes de la enfermedad mental y sus consecuencias sociales, económicas, culturales y biológicas.

La rotación anteriormente mencionada forma parte del Módulo 5: Intervención de Enfermería con la Adultez en Procesos Mórbidos de Salud, mismo que está incluido dentro del plan de estudios de la carrera de Licenciatura en Enfermería, impartida en la Universidad de Costa Rica.

Como parte del enfoque de promoción de la salud y el fomento de estilos de vida saludable, es que se torna importante trabajar en la comunidad, con el fin de abordar la problemática de salud mental y romper así el enfoque tradicional de la psiquiatría el cual se relaciona principalmente con la atención institucionalizada.

Por lo presentado anteriormente y según los propósitos planteados, se hace necesario contar con un sustento teórico que constituya la guía para analizar las experiencias vividas.

\section{Marco de Referencia}

Los seres humanos estamos en constante búsqueda del bienestar biológico, psicosocial, económico, ambiental, y en su consecución somos partícipes activos 
de la construcción de nuestra salud. Cuando existe alguna alteración en una de estas dimensiones puede modificarse la calidad de vida de la persona, como es el caso del trastorno mental.

Dada estas repercusiones, la Organización de la Naciones Unidas (2002), evidencia preocupación en la problemática de la salud mental, esto al afirmar la relación entre la salud y el desarrollo de las Américas, mostrando así las consecuencias económicas de las enfermedades mentales en la región. Dicha organización ejemplifica que "los trastornos psiquiátricos causan 1000 millones de días productivos perdidos al año" (OPS, 2002: p.197). Con lo anterior se muestra el impacto laboral de estos padecimientos, los cuales tienen implicaciones en el estilo de vida de la persona.

Costa Rica como parte de la región de las Américas se encuentra inmersa en esta realidad; según la Situación Actual de la Salud Mental en Costa Rica (2004), hay factores que pueden desencadenar el deterioro de la misma, dentro de ellos se pueden mencionar: la pobreza, la urbanización desordenada, las condiciones de trabajo desfavorables, la violencia, los desastres naturales, situaciones de crisis y aspectos biológicos como la edad, trastornos mentales y trastornos del desarrollo. Por lo anterior se hace evidente la relación entre condición de vida y la problemática en salud mental que se presenta a nivel nacional.

Así mismo Guinsberg (1985) menciona que muchas de las causas de los trastornos mentales no se justifican por una lesión orgánica, más bien tienen relación con los factores sociales mencionados anteriormente, por lo que se considera a la sociedad responsable y corresponsable de las perturbaciones mentales.

Estos lineamientos llevan a definir la enfermedad mental según la Asociación Americana de Enfermeras (1994), como

“una alteración en los
pensamientos o en el estado de
ánimo que rausa
comportamientory con
adaptación regativa,
incapacidad para hacer frente
a las tensiones normales y
deterioro en el desempeño o
todos a la vez." (citado por
O'Brien 2001: p.27).

En estas situaciones de alteración del estado de salud mental, se debe destacar la importancia que tiene atender los estados elevados de ansiedad, así como las consecuencias derivadas de trastornos del afecto. Ansiedad y depresión corresponden a dos de los mayores problemas de salud mental según el perfil epidemiológico en Costa Rica.

Para O’ Brien (2001) y Bobes (1996) se debe entender que la ansiedad es un mecanismo de defensa al cual el organismo acude en determinadas situaciones que implican una reacción de alerta; no obstante, adquiere su estado patológico cuando interfiere en el funcionamiento de la persona. Al respecto O’Brien (2001) señala que la intervención de enfermería hacia estos individuos debe ir enfocada a la comprensión y muestras de interés hacia la persona y su familia.

La depresión se caracteriza clínicamente por la presencia persistente y dominante 
de un sentimiento negativo sobre el propio funcionamiento, el autoconcepto y el futuro, predominando una sensación de culpa y baja autoestima. Esto se manifiesta en una serie de dificultades sociales que tienen como eje la pérdida del interés por las actividades cotidianas. Es por ello que el Consejo Internacional de Enfermeras (CIE) (2002) menciona que nuestra disciplina favorece la promoción de la salud mental, la prevención de la enfermedad, los cuidados, el tratamiento y la rehabilitación, además de representar un gran apoyo a la familia y la comunidad, con esto se enfatiza el abordaje en la atención primaria.

En nuestro país el Ministerio de Salud ha implementado estrategias para la atención en salud mental, tal y como lo manifiesta la OPS (2004) al recalcar la importancia de descentralizar la atención psiquiátrica hacia el modelo comunitario, garantizar el cumplimiento del derecho de la población a la salud mental y finalmente incluir a ésta dentro del sistema de vigilancia de salud.

La promoción de la salud va acorde con la protección de los derechos de las personas con trastornos mentales, por lo que el modo y el estilo de vida deben representar el eje central de acción. El Ministerio de Salud en el Plan Nacional de Salud Mental (2004-2010) menciona que se debe educar en todos los aspectos de la vida humana a saber el autocuidado de la salud, la alimentación saludable, la educación para actividades recreativas y deportivas, la importancia de las relaciones interpersonales y el contacto emocional, el fomento de grupos de apoyo y la interacción social, entre otras. Todos estos aspectos son puntos a contemplar en la intervención de enfermería; basándose en lo propuesto por Hildergard Peplau (1990: p.191) al afirmar que el enfermero(a) "tiene la responsabilidad de ayudar al paciente a mejorar sus habilidades para afrontar los problemas más que enseñarles a solucionarlos". Lo anterior evidencia que la educación en salud proporcionada por el y la profesional en enfermería, brinda herramientas para potenciar su bienestar y fomentar una mejor calidad de vida.

De acuerdo con lo anterior, muchos países han incorporado en sus sistemas de salud la práctica de la salud mental comunitaria (SMC). Esto se evidencia en el artículo "Principios de salud mental comunitaria" del Dr. Hugo Cohen (2001) quien describe y relaciona los contextos de dichas regiones con los ocho principios que fundamentan esta filosofía.

Así mismo, Chávez y colaboradores (2006) realizaron una sistematización de un proceso terapéutico desarrollado con cuatro sistemas familiares que cuentan con un miembro que presenta una alteración mental. De este estudio se concluye que el trastorno mental en un integrante de la familia, afecta la dinámica de la misma, así como también se puede notar que debido a condiciones de género son las mujeres quienes en la mayoría de los casos desempeñan el rol de cuidadoras. Aunado a lo anterior, las familias no disponen de adecuados mecanismos de afrontamiento en cuanto a cuidado y atención del individuo con problemas mentales, haciéndose fundamental la educación brindada por el y la profesional en enfermería, con el fin de suplir tales necesidades.

\section{Materiales y Métodos}

Para la elaboración de la investigación se utilizó como metodología la 
sistematización, de experiencias mencionada por Jara (1994), la cual consta de cinco pasos:

a) El punto de partida: consiste partir de la propia práctica, por lo que se debe haber participado en la experiencia y poder contar con registros claros $\mathrm{y}$ precisos de ello.

b) Preguntas iniciales: se da la organización en tres ubicaciones esenciales: la definición del objetivo (la utilidad, el producto o el resultado que se espera obtener de la sistematización), la delimitación del objeto o las experiencias concretas en lugar y tiempo, y la precisión del eje de sistematización, que consiste en el enfoque, el hilo conductor de todos los aspectos de esas experiencias, acorde con el objeto y objetivo de estudio.

c) Recuperación del proceso vivido: se reconstruye la historia permitiendo una visión general y cronológica de los principales acontecimientos o etapas que han sucedido a lo largo de la experiencia. Se avanza hacia el orden y clasificación de la información, al ubicar los distintos componentes del proceso, tomando en cuenta los ejes de sistematización.

d) La reflexión de fondo, ¿Por qué pasó lo que pasó?: es la interpretación crítica del proceso vivido, se da el análisis, síntesis e interpretación crítica del proceso.

e) Los puntos de llegada: es la formulación de las conclusiones tanto teóricas como prácticas, según el eje de sistematización y dando respuesta a los objetivos planteados y por último se hace referencia a la comunicación de los aprendizajes.

La base de esta sistematización consistió en la experiencia vivida por un grupo de 7 estudiantes de enfermería del cuarto año de la carrera, al realizar la rotación de Procesos Mentales Mórbidos dentro del Módulo de Adultez Mórbida, esto durante el mes de agosto del año 2006. Esta práctica se organizó en coordinación con un Centro de Salud del Área Metropolitana.

La escogencia de esta población se hizo por recomendación de las médicas encargadas del Centro de Salud, y la asignación de las familias fue hecha al azar por las docentes del curso. Los subgrupos de trabajo se realizaron a conveniencia de las estudiantes en dos parejas y un trío; la intervención se realizó en tres personas con procesos mentales mórbidos en edades comprendidas entre los 43 y 77 años, siendo dos de ellas mujeres y un varón. Se hizo bajo la técnica de Estudio de Caso, la cual consiste según Duarte (1997) en: "la investigación descriptiva de carácter intensivo de una persona o fenómeno a través de un seguimiento y evaluación sistemática de las observaciones, y sirve para obtener una descripción completa de los aspectos típicos de una persona”, es así como se busca abarcar a cada individuo en todas sus dimensiones y necesidades.

Como medio para valorar a las personas, se utilizaron los siguientes instrumentos:

Revisión del Expediente Clínico: según las referencias halladas en el expediente, se hizo una indagación de las anotaciones hechas en las citas de Psiquiatría pertinentes a un Hospital del 
Área Metropolitana donde acudieron las personas del estudio de caso, dado que las valoraciones médicas requerían de esta especialidad.

Genograma: Madaleno

menciona que esta estrategia brinda una representación gráfica de las relaciones familiares, incluye patrones de enfermedad, problemas psicosociales y factores protectores en la dinámica familiar.

Apgar Familiar: según Rodríguez (2004) es una medición de cinco aspectos claves de la funcionalidad familiar. Permite detectar la percepción individual de cada miembro de la familia.

Historial de Enfermería: Océano (2002) señala que es una entrevista, la cual está estructurada según las distintas dimensiones de la persona: psicosocial, biológica, económica y ecológica.

Cuestionario General de Salud de Goldberg: según García (1998, citado por Meza, 2006) es un cuestionario autoadministrado que identifica la severidad de los trastornos psiquiátricos dirigido a la situación actual de la persona, enfocándose en su estado de bienestar general.

Mini Examen Mental: de acuerdo a Meza (2006) es usado para valorar el estado mental de la persona, en el cual se busca conocer el contexto que gira alrededor del trastorno mental, así cómo y cuánto ha afectado el mismo las capacidades de la persona que lo padece. Ahora bien los aspectos mencionados anteriormente, se valoran con la realización de la entrevista o historial de enfermería, el cual fue definido previamente. Para la sistematización se establecieron los siguientes ejes:

Los ejes están
definidos por la
relación entre la
valoración de la
persona y la familia y
las situaciones de
enfermería
identificadas, así como
las acciones que se
implementaron.

Atención de Enfermería

\section{Ejes de Sistematización}

\section{Evaluación de la situación de salud de la persona}

El primer estudio de caso, se realizó a N. G. P. una señora adulta mayor de 77 años, de nacionalidad nicaragüense. Actualmente no desempeña ninguna ocupación. Padece de depresión desde hace aproximadamente tres años, además sufre de hipertensión arterial, hipotiroidismo, dolores óseos y hace más de 25 años sufrió un infarto.

Entre los hallazgos más importantes encontrados en el genograma destaca que: su abuela padeció de "tristeza", lo cual es un antecedente de trastornos afectivos, ya que tanto la usuaria como su hija menor padecen de depresión. La relación de la señora con sus padres era buena. Tiene dos hermanos y dos hermanas. Su esposo murió hace 26 años; la relación entre ambos fue muy estrecha, por lo que este fallecimiento fue un acontecimiento muy doloroso para la usuaria. De esta unión nacieron dos hijas (61 años y 56 años de edad). Las patologías que se manifiestan con más fuerza en la familia de la señora son: hipertensión arterial, enfermedades cardíacas y trastornos depresivos. 
En cuanto al mini examen mental, se encuentra que la apariencia física de la usuaria está descuidada. Durante las visitas se le observó en ropa de dormir y sin bañarse, además la persona comenta que no es tan vanidosa como antes. Se percibe decaída, con actitudes pasivas, un gesto de resignación, con caminar despacio, utiliza poco lenguaje corporal cuando habla y su lenguaje verbal es lento y pausado.

En lo referente al historial de enfermería, N.G.P. se describe como una persona sensible, independiente y satisfecha con su vida. Sus relaciones familiares son estrechas y las sociales están restringidas. Entre las situaciones que le generan más estrés están las preocupaciones por conflictos de sus familiares. Es sedentaria, fumadora, sus horarios de sueño son prolongados y presenta hábitos alimenticios deficientes. En lo referente a su historia socioeconómica posee vivienda propia, la cual está en buenas condiciones, con accesibilidad a los servicios básicos.

Según la revisión del expediente clínico la usuaria asiste al EBAIS para control de la hipertensión arterial. Los motivos más frecuentes de consulta son dolores musculares y óseos. Se le ha brindado tratamiento farmacológico para la depresión, el hipotiroidismo y la hipertensión arterial.

El segundo estudio de caso fue realizado con la señora IB, adulta media de 43 años, es la primera de dos hermanos, su madre tiene 68 años y es con quien posee la mejor relación, su padre falleció cuando ella tenía 10 años de edad, de un infarto. Comenta que la relación más conflictiva y distante es con su hermano. Como antecedentes familiares se encuentra la hipertensión y la diabetes.
Ella vive con sus tres hijas de 17, 15 y 10 años, se separó de su esposo hace aproximadamente un año por problemas de agresión intrafamiliar, refiere poseer una relación distante con sus hijas. Las tres muchachas estudian en colegios privados. La segunda de ellas padece rinitis alérgica, tiene un ovario poliquístico y según refiere la madre, se sospecha que padece lupus, la hermana menor también padece rinitis.

Referente al historial de enfermería la señora cuenta con casa propia en buenas condiciones, la única fuente de ingresos es la pensión que recibe, sin embargo comenta que esta no le es suficiente para sus gastos, afirma que le agrada el lugar donde habita y comenta que es seguro. La adulta dice sentirse deprimida, triste y frustrada; además se describe como una persona con carácter débil; comenta que la forma en que expresa sus sentimientos es por medio del llanto. Para disminuir el estrés escucha música y pertenece a un grupo de oración; sin embargo en este grupo no comparte sus problemas. Como molestias físicas destacan los problemas visuales, la hipertensión y las dificultades para dormir, sin embargo cuenta con adecuados hábitos alimenticios.

Dentro de los aspectos sobresalientes en la evaluación mental se destaca la presencia de pensamiento saltón, esto es que cambia súbitamente de un tema a otro por lo que es difícil mantener la coherencia en la conversación.

Según el cuestionario de Goldberg, afirma que se siente bastante peor de salud, manifiesta falta de ánimo y no se siente feliz, además de que sus niveles de concentración han decaído, comenta que siente que realiza las cosas peor de 
como lo hacía antes. También se siente incapaz de tomar decisiones $y$ ha manifestado ideas suicidas.

Según el APGAR familiar, la adulta cataloga su familia como disfuncional, mientras que sus hijas mayores piensan que es medianamente funcional y su hija menor considera que su familia es altamente funcional.

El tercer estudio de caso se llevó a cabo con un adulto de 54 años de edad, C.P.B, Master en Ingeniería Civil. Soltero, no tiene hijos y vive solo. Presenta antecedentes familiares de cáncer gástrico, próstata e infarto agudo de miocardio, problemas cardiovasculares y renales, además colesterol alto. Entre los padecimientos personales de C.P.B están la hipercolesterolemia, gastritis, desviación en el duodeno y traumatismo en el miembro superior derecho.

En la revisión del expediente se encuentra que desde hace un mes posee una incapacidad laboral por un accidente vascular cerebral(AVC) trombótico, el cual le dejó secuelas de afasia y vértigo secundario, que han tenido un progreso optimo, sin embargo dicha persona refiere no estar en capacidades o condiciones para asistir al trabajo debido a que los medicamentos que esta tomando le producen somnolencia, además todavía no está seguro de estar totalmente recuperado por lo que no podría dar el rendimiento laboral óptimo. Sin embargo presenta una ansiedad relacionada con la dependencia hacia el trabajo, por lo que estando en su casa sigue ejecutando actividades laborales a pesar de estar con incapacidad. Otros de los diagnósticos por los cuales fue referido del EBAIS son: depresión, conductas compulsivas y adicción al trabajo. Se pudo constatar que se encuentra en tratamiento con psicología y neurología, además de control del colesterol, las consecuencias del accidente vascular cerebral y la ansiedad.

Según el Genograma las relaciones interpersonales con su familia son conflictivas y distantes, con quien mantiene la relación mayormente significativa es con el sobrino de 17 años, por lo que la relación que tuvimos con la familia fue poca.

De acuerdo al historial de enfermería presenta relaciones interpersonales poco satisfactorias, pues menciona sentirse inseguro y con necesidad de afecto. Posee un patrón de comunicación poco funcional, un inadecuado manejo del estrés y alteraciones del patrón del sueño. Uno de los factores protectores más sobresalientes es la adecuada nutrición.

\section{Situaciones de enfermería encontradas}

Una vez analizados los datos registrados durante la valoración de enfermería se deducen las situaciones de enfermería que van orientar el plan de intervención, algunas de estas situaciones son las siguientes:

\section{Sobre los estilos de vida.}

1. Alteración del patrón del sueño.

2. Alto riesgo de padecer enfermedades crónicodegenerativas.

3. Alteración de los niveles de presión arterial.

4. Inadecuados hábitos alimenticios.

5. Reducción de la actividad física.

En lo referente a los estilos de vida se observó la similitud de los tres estudios 
de caso, lo que evidencia problemas de autocuidado que se pueden relacionar esencialmente con la actividad-descanso, alimentación y predisposición a enfermedades crónicas y degenerativas. Así mismo dichos estados reflejan un desinterés preponderante en las actividades cotidianas, pues derivan toda su energía al proceso mental mórbido que experimentan.

\section{Sobre la enfermedad mental.}

1. Alteración en el estado anímico.

2. Déficit de conocimiento sobre la enfermedad.

3. Déficit de conocimiento sobre el tratamiento farmacológico.

De acuerdo con lo anterior, se puede notar que existen carencias educativas por parte del profesional de salud en lo referente a la información que se le brinda a la persona con respecto a la enfermedad y al tratamiento que recibe. A pesar de que los factores desencadenantes del proceso mental son distintos en los casos estudiados, sus manifestaciones se basan en la disfunción del estado anímico, afectando el desempeño de la persona en diversos ámbitos de su vida.

\section{Sobre la convivencia.}

1. Dificultades generadas por una baja autoestima.

2. Inadecuada comunicación familiar.

3. Aislamiento social.

4. Relaciones interpersonales poco satisfactorias.

La soledad que estas personas experimentan dada su enfermedad mental, les lleva a inhibir de manera significativa su relación con el medio, exacerbando así el cuadro psiquiátrico manifestado. Esto se refuerza con el temor y poca seguridad en si mismos, pues no aprovechan sus fortalezas, aunado a esto el proceso de socialización se ve influenciado por los esteriotipos culturales acerca de los trastornos mentales.

\section{Acciones de enfermería}

La intervención de enfermería se basó en tres medidas fundamentales: la educación, la orientación y la comprensión empática con el propósito de establecer una relación de confianza.

- Educación y orientación: referente a la enfermedad mental $y$ otras patologías presentadas, el tratamiento de la misma, los estilos de vida saludable, formas de desarrollar relaciones interpersonales efectivas.

- Consejerías: brindando opciones para la sensibilización y el desarrollo de actitudes positivas hacia el control médico y psicológico, establecimiento de alianzas con grupos de apoyo, así como fomentar el autocuidado.

- Comprensión empática para establecer una relación de confianza: facilitando la expresión de sentimientos, externar dudas acerca de su situación de vida, fomentando una comunicación asertiva, la escucha activa y dando apoyo continuo.

\section{Análisis de los resultados}

\section{Recuperación del proceso vivido}

Como se mencionó anteriormente, dentro del Módulo de Intervención de Enfermería con la Adultez Mórbida, existe una Rotación de Psiquiatría con una duración de un mes, en la misma se 
pretende obtener los conocimientos $\mathrm{y}$ ciertas habilidades en la atención que se le brinda a una persona con procesos mentales mórbidos.

Con el fin de conocer la situación actual en la salud mental costarricense, se realizó una visita al Hospital Nacional Psiquiátrico el día 22 de agosto. A pesar de tener ciertos temores por los tabúes que maneja la sociedad, esta visita generó gran conciencia y un sentido de humanización hacia estas personas que por su condición de salud se les ve comúnmente como seres inferiores. A pesar de que la infraestructura es un medio que inspira tranquilidad y es adecuada, se nota el desaprovechamiento de los recursos, ya que la rehabilitación de estas personas no se realiza con la totalidad de estos medios.

Las personas internadas están divididas en dos sectores, uno para hombres y otro para mujeres, cada uno se agrupa por pabellones según los diagnósticos de ingreso, esto para nosotras fue una buena decisión, ya que la convivencia de las personas no se ve alterada por conductas exacerbadas que generan otras patologías mentales. La presencia de abundantes zonas verdes permite a los individuos tener una relación cercana con la naturaleza, lo que para nosotras significó un punto a favor, ya que este contacto los acerca a la realidad y a la vida natural.

En lo que concierne a los estudios de caso, se realizaron en dos de ellos, dos visitas y en el restante, solamente una, ello no implicó obviar los objetivos determinados para su realización, a saber:

- Identificar la percepción de la familia sobre la conducta de la persona en cuanto a sentimientos, expectativas $\mathrm{y}$ dudas.

- Identificar la percepción de la familia sobre el tratamiento de la persona en cuanto a efectos de los medicamentos, manejo adecuado, grado de aceptación y efectividad.

- Identificar la percepción de la persona sobre su situación de salud, familiar, de tratamiento y rehabilitación psicosocial.

\section{Caso I}

\section{Sobre la práctica misma:}

En el desarrollo de esta práctica, cabe destacar la dificultad que se presentó en uno de los casos. El mismo fue asignado por las profesoras de la rotación de psiquiatría en coordinación con el personal médico del centro de salud. En primera instancia se iba a tratar con una persona, a la cual se contactó en tres ocasiones vía telefónica con el fin de explicar los propósitos de la intervención de enfermería y obtener el consentimiento para esta. Así mismo se llevó a cabo la revisión del expediente clínico, para conocer detalles importantes sobre la historia de salud de la persona. Sin embargo, no se pudo establecer un encuentro personal con ella, ya que ésta postergó dos veces las citas y en la tercera llamada mencionó que no estaba interesada en llevar a cabo la intervención. Esto último generó ansiedad e incertidumbre por parte de las estudiantes, debido a que dificultó el comienzo de la práctica.

Con el propósito de contrarrestar la anterior situación, se acordó asignar un nuevo caso, se realizó una llamada telefónica, en la cual se coordinó la reunión y se obtuvo la aprobación de la usuaria. Luego se procedió a la revisión 
del expediente clínico; lo que permitió orientar el primer acercamiento con la persona.

Sobre cada persona y familia de acuerdo a los ejes de sistematización:

La primera visita se llevó a cabo el día 25 de agosto del año 2006, tuvo una duración de una hora y media aproximadamente. El objetivo de este primer contacto fue valorar la situación de salud de la persona y a partir de esto acordar con ella los elementos que se iban a tratar en el próximo encuentro.

Al llegar a la residencia de la señora, esta se mostró muy amable y atenta. Un aspecto destacable es que estuviera en ropa de dormir y sin bañarse al medio día. Posteriormente, se dio inicio a la entrevista, en la cual comentó situaciones importantes en su historia de vida como la muerte de sus padres y hermanos, así como el fallecimiento de su esposo, el cual le provocó nostalgia e incluso hizo que sus ojos se llenaran de lágrimas. Este acontecimiento originó en las estudiantes un sentimiento de empatía. Además describió entre otras cosas, como eran las relaciones con sus familiares y amigas, lo que más le gusta hacer y el lazo afectivo con su mascota.

De la misma manera, la señora expresó sus preocupaciones acerca de su condición de salud, mencionó la evolución de las enfermedades y el tratamiento utilizado para estas. También habló sobre su percepción y la de su familia acerca del trastorno mental. $\mathrm{Al}$ finalizar la reunión, se establecieron los asuntos que se iban a abarcar en la segunda visita.

Ahora bien, el segundo encuentro se realizó el día 28 de agosto del año 2006 y duró igualmente una hora y media. En un principio, se creyó que no se iba a poder efectuar, debido a que la señora aún dormía y no atendía al llamado. Eventualmente, después de más de quince minutos de espera, salió a abrir la puerta. La señora presentaba la misma apariencia física de la visita anterior.

El tiempo de esta visita se empleó para implementar las acciones planeadas con respecto a las situaciones de enfermería encontradas. Dichas acciones se enfocaron en la educación sobre estilos de vida saludable, el trastorno depresivo y el tratamiento farmacológico.

La señora mostró interés y colaboración durante la sesión, participó e hizo preguntas. Posteriormente se llevó a cabo la evaluación de la información brindada, la cual reflejó un grado satisfactorio de compresión; con lo que dio fin a la intervención.

Este proceso fue enriquecedor a nivel académico porque permitió la intervención de enfermería en la promoción de la salud dirigida a una persona con procesos mentales mórbidos y con esto se pusieron en práctica los conocimientos adquiridos durante la rotación y los años en la carrera. De igual forma fue valioso a nivel personal, por que se estableció una relación empática con la usuaria, que a la vez facilitó el desarrollo de la atención proporcionada. Además, constituyó una oportunidad para romper prejuicios y adoptar una perspectiva diferente respecto a los trastornos mentales y el abordaje de los mismos.

\section{Caso II}

Sobre la práctica misma: 
Bajo la coordinación previa de las profesoras a cargo y las médicas del Centro de Salud, nos asignaron el caso de una adulta media de 43 años de edad, diagnosticada con depresión severa y sobrevivencia de violencia intrafamiliar, la misma se consideró pertinente para que se cumplieran los objetivos de intervención.

De esta manera se procedió a realizar una llamada telefónica con el fin de comentarle a la persona los fines de la visita domiciliaria que realizaríamos como estudiantes de enfermería. Una vez obtenido su consentimiento evidenciando gran anuencia, se concretó la cita para el encuentro en el hogar y se procedió a la revisión del expediente clínico. Durante esta, se prestó especial atención a los registros concernientes a la salud mental, también se tomaron en cuenta otros los problemas de salud para abordar a la persona integralmente.

La primera visita fue concretada el día 24 de agosto a la 1 de la tarde. A la hora establecida se llegó al lugar y se llamó a la puerta de la casa en tres ocasiones sin obtener respuesta, ante esta situación, se abandonó el lugar. Esto generó en nosotras una gran incertidumbre por no saber que había ocurrido con la persona; además de mucha ansiedad.

Después de reprogramar la visita y hablar previamente con la persona, nos comentó que no podría atendernos, así que se excusó puesto que tenía un imprevisto. Ante esta situación, se decidió consultar a la persona y preguntarle nuevamente si estaba dispuesta a atendernos, rescatando la importancia de la información que se le deseaba brindar. De esta manera, la persona empezó a comentar un poco de su historia de vida y de la situación mental por la que estaba pasando, expresó vía telefónica que en muchas ocasiones había deseado llegar al suicidio. Ante el inconveniente de tratar este tema por teléfono, se le consultó si deseaba hablar personalmente, ante lo cual estuvo anuente y nuevamente se programó la visita par 28 de agosto.

Partiendo de lo comentado por I.B. vía telefónica, decidimos preparar el material didáctico que utilizaríamos para brindar educación.

\section{Sobre cada persona y familia según los ejes de sistematización}

Durante la conversación, nos comentó su situación mental, expresando que vivía una depresión. Comentó que se sentía lo triste y sola y que a pesar de vivir con sus hijas, la comunicación entre ellas ha fallado mucho y no hablan sobre sus sentimientos. A pesar de que ha recibido el apoyo de su madre, considera que se ha alejado un poco de ella porque ésta le reclama que constantemente esté llorando y lo que ella llama "su actitud de derrota". Esto le ha generado tristeza y conflicto interno, ya que dice sentirse débil y muy inferior a su madre, por lo que comenta que desea parecerse a ella en su fuerza y coraje para salir adelante.

Ante esta situación, nos dimos a la tarea de reforzar sus actitudes positivas, sus fortalezas y lo valioso de su persona, procurando que ella por sí misma descubriera que por ser mujer y madre, ya cumple un papel indispensable en su propia vida, para de esta forma mejorar su autoestima.

Los comentarios emitidos inicialmente por la persona, nos afligieron mucho $\mathrm{y}$ nos dejaron contrariadas al ver que 
cuando más necesita de apoyo familiar es cuanto más sola está.

En otro momento de la visita, se prosiguió a consultar sobre los medicamentos que tiene prescritos como parte del tratamiento de las enfermedades que le aquejan $y$ se prosiguió a dar educación sobre este tema.

Por otra parte, se hicieron recomendaciones ya que se evidenció que I.B. se estaba aislando de sus amistades y del grupo comunitario al que pertenecía. Ella mostró duda ante la realización de actividades alternativas, lo que nos generó cierta incertidumbre de si en realidad ella las implementaría.

\section{Caso III}

\section{Sobre la práctica misma}

El viernes 18 de agosto, fue la visita al centro de salud, esta se realizó en la tarde, experimentamos una sensación de tranquilidad por la orientación que las profesoras brindaron. Se procedió a coordinar la aprobación de la intervención de enfermería vía telefónica, con la cual el señor C.P.B. accedió de modo muy anuente e interesado, posteriormente nos dimos a la tarea de revisar el expediente clínico.

Esta experiencia fue muy satisfactoria, nos permitió fortalecer habilidades tanto en el aspecto profesional como el personal, favoreciendo el crecimiento y la confianza en nuestro trabajo, además de que nos reforzó el conocimiento adquirido en años anteriores.

Sobre cada persona y familia de acuerdo a los ejes de sistematización

La primera visita se realizó el martes 22 de agosto, esta se enfocó en una fase de valoración y con ello obtener un panorama más amplio y personalizado para saber como abordar a la persona.

El señor nos recibió en su casa, al ingresar lo primero que se vio en la sala fueron tres monitores, lo que nos dejó sorprendidas y dejaba claro que el señor trabajaba mucho en ellas. Al observar su hogar detallamos que no tenía muebles de sala (sofás), por lo que nos dio la impresión de que no hay espacios para establecer un ambiente familiar cómodo y agradable, igualmente nos dimos cuenta de que vivía sólo, que las relaciones con sus familiares eran conflictivas y distantes a pesar de la cercanía geográfica.

Por consiguiente, nos comentó que había tratado de ordenar la casa un poco, y le explicamos en lo que consistía esta primera visita. Él se mostró muy atento, sin embargo, fue notable la ansiedad que le producían algunas preguntas relacionadas con su autopercepción, las cuales conforme avanzaba la entrevista fueron respondiendo.

La conversación se extendió más de lo pensado, en un tiempo aproximado de 3 horas, el señor C.P.B. se mostró muy expresivo, conversaba de manera fluida acerca de los temas tratados, lo cual permitió que ahondáramos en su historia de vida.

La segunda visita realizada el 28 de agosto fue amplia, consistió en dar educación según las necesidades expuestas en la reunión anterior. En esta igualmente nos recibió muy bien y atento a cada punto a tratar. En esta visita se pudo profundizar en su historia de vida, debido a que expresó resentimientos hacia las mujeres a lo 
largo de su historia, dentro de los recuerdos de mayor relevancia para esta persona, se encuentran: el fallecimiento de su padre cuando él tenía 3 años, menciona que su madre fue una persona fría que no le demostró el cariño y afecto que este como niño merecía, en la escuela recuerda a una directora autoritaria que en más de una ocasión lo agredió, además la hermana también lo agredía, manifiesta que casi no tuvo muchos amigos en la escuela, ni en el colegio, por lo que expresa sentimientos de soledad y dificultad para establecer relaciones interpersonales significativas. Ante el hecho de realizar la última visita, el señor nos comentó que le agradó mucho poder compartir con nosotras, expresar sus sentimientos, dudas y situaciones, por lo que nos generó una sensación de alivio y satisfacción en la labor cumplida.

\section{Reflexión de fondo}

En este momento de la sistematización nos planteamos las siguientes interrogantes que nos permitieron iniciar el análisis:

- ¿Qué relación tienen las alteraciones en el estilo de vida de las personas con la enfermedad mental?

- ¿Cuáles son las condiciones económicas, ecológicas, psicosociales que coinciden en los tres casos y su relación con los trastornos mentales?

- ¿Cuáles son las dificultades en la atención de enfermería hacia las personas con trastorno mental en el primer nivel de atención?

Para dar respuesta a las preguntas anteriormente formuladas, se realiza el posterior análisis:
Existe relación recíproca evidente entre los estilos de vida de las personas y su enfermedad mental, esta relación va a estar determinada por los factores de riesgo o factores protectores existentes, pues las alteraciones en algunos de los aspectos producen efectos desfavorables. Como lo afirma Guinsberg (1985) hay factores que pueden influir en que una persona padezca un problema mental, tienen relevancia tanto los aspectos biológicos como los factores psicosociales, entre estos se destacan: la falta de cariño de los padres, el conflicto en las relaciones de pareja, en las relaciones laborales y la carencia de afecto y relaciones interpersonales satisfactorias.

Lo anterior pone en evidencia que muchos de los estilos de vida manifestados en los estudios de caso analizados, se relacionan con la incidencia de una enfermedad mental.

Las familias de los tres casos estudiados son uniparentales, ocasionado por situaciones como la muerte de la pareja o conflictos conyugales. Esto evidencia que puede existir una relación entre el desarrollo de dificultades mentales y la estructura de la familia; según lo descrito por Adis Castro "la mayor prevalencia de problemas psiquiátricos tiende a ocurrir en personas solteras, en primer lugar, y entre divorciados, en segundo lugar" (Adis Castro, 1992:45). Por consiguiente se afirma que hay una fuerte relación entre la presencia de problemas mentales con estas dos categorías de estado civil.

Con respecto a las similitudes, en los tres casos se evidencia que corresponden a la clase media alta, es notable que quien posee sus ingresos por cuenta propia es 
el hombre, y las dos mujeres son dependientes de otras personas, esto por razones de pensión económica, por lo que es sobresaliente la influencia de género en la dedicación a actividades laborales. Estas diferencias de género, no sólo están relacionadas con el patriarcado en la sociedad costarricense, sino también por la poca importancia que se le da al quehacer de las mujeres, mucho de ello tiene que ver con lo afirmado por Daskal (1990) al resaltar que la vida cotidiana de las mujeres abarca insignificancias como el trabajo doméstico, su invisibilización y desvalorización.

Según el entorno ecológico, las tres personas y sus familias conviven en un ambiente agradable, tranquilo, no son notables factores de riesgos como vectores biológicos ni delincuencia que afecten su seguridad. En cuanto a su entorno inmediato, se refleja que las viviendas cuentan con los servicios básicos; es destacable que en dos de los casos consideramos que la residencia está desordenada.

De acuerdo a las evidencias recogidas en la valoración, las personas cuentan con recursos para realizar el autocuidado, sin embargo sus enfermedades han acaparado su atención dejando de lado el cuidado personal, lo que agrava su situación de salud.

Referente al aspecto psicosocial es característico en los tres casos un autoconcepto donde esta presente la idea de debilidad, los sentimientos de soledad y el aislamiento social. En el caso II y III se evidencia carencia de apoyo familiar y las relaciones entre estos son conflictivas y distantes.
Lo anterior se relaciona con un estudio realizado por Adis Castro (1992), en el que destaca que dos factores: "la aceptación de tener conflictos interpersonales" y "la aceptación de tener sentimientos de soledad" se encuentran directamente asociados a la presencia de sintomatología psiquiátrica. Además el autor menciona que los factores relacionados con el sentimiento de soledad se asocian con la timidez, la carencia de familiares y amigos que den apoyo, y el tener que relacionarse con personas con las que no se comparte ni interés ni afectos, y que tienen otras preferencias y actividades recreativas.

Agregado a esto, tenemos que las técnicas para el manejo del estrés implementadas por las personas en los tres casos son poco efectivas, ya que usualmente estas fomentan el estado de alteración mental, manteniendo y exacerbando el trastorno que presentan. Según O`Brien (2001), las capacidades de enfrentamiento son mecanismos que las personas utilizan para manejar factores estresantes internos y externos; identificar la característica de afrontamiento ayuda a determinar las medidas que pueden emplear en una situación de crisis.

Todo lo anterior está relacionado con la dificultad que encuentran las personas en cuanto a la atención que reciben por parte del Sistema de Salud en Costa Rica en el primer nivel atención.

La Caja Costarricense del Seguro Social (2000) destaca que dicho nivel carece de un programa de salud mental y de apoyo sistemático en cuanto a capacitación, promoción de la salud mental, rehabilitación psicosocial, salud mental escolar, organización de grupos de 
autoayuda, asesorías a organizaciones no gubernamentales y comunales entre otros, por otra parte dicha institución enfatiza en las deficiencias que encierra la falta de respeto y cumplimiento de los derechos humanos hacia la persona con trastorno mental.

Desde esta panorámica se evidencia que en el centro de salud donde acuden las personas de dichos estudios de caso, las pautas expuestas anteriormente se cumplen, pues no respetan las normas de atención, al trasgredir el derecho a la información sobre su padecimiento y al no contar con medidas necesarias para dar una adecuada rehabilitación psicosocial, orientando sus acciones al aspecto farmacológico, desde un enfoque biomédico.

Es por ello que de acuerdo con lo mencionado por la OMS (2001) se hace necesaria la reinversión de recursos que favorezcan la descentralización, brindando un mayor aporte económico a la atención primaria y a la promoción de la salud mental.

Esto deja claro que el modelo de atención en salud mental vigente según la CCSS (2000) en Costa Rica mantiene una estructura centralizada en hospitales del tercer nivel enfatizando la atención psiquiatrica directa a trastornos de mayor impacto social, como es la esquizofrenia, el trastorno bipolar, entre otros, dejando de lado la atención de trastornos menores que igualmente presentan consecuencias graves para la sociedad y para el individuo, además que su atención se da mayormente en el momento en que la persona presenta estados descompensados.

\section{Conclusiones}

\section{Puntos de llegada}

Es evidente la relación significativa entre los inadecuados estilos de vida y la enfermedad mental, ya que los factores de riesgo tienen mayor prevalencia que los factores protectores, poniendo en desventaja a estas personas, ya que estas presentan otras patologías como las enfermedades crónicas degenerativas, que dificultan el proceso de rehabilitación.

Las relaciones interpersonales poco efectivas pueden ser precursoras $y$ agravantes de la enfermedad mental en las personas, pues presentan disfunciones en la comunicación, en la expresión de afecto y sentimientos hacia los demás, lo que imposibilita la búsqueda de apoyo y una rehabilitación óptima.

La orientación y la educación de la persona sobre su trastorno mental permiten mayor empoderamiento que va a favorecer el mantenimiento, cuidado y fortalecimiento de la salud, beneficiando la calidad de vida.

Se destaca la necesidad de contar con personal capacitado en el primer nivel de atención, además de recursos para la detención temprana de trastornos mentales, así mismo, que se dediquen esfuerzos orientados a la promoción de la salud mental con una visión interdisiplinaria e intersectorial.

Las situaciones de vida que experimenta la sociedad costarricense, representada en los casos estudiados, reflejan que esta no escapa de la realidad mundial, ya que la salud mental debe formar parte de los pilares de promoción de la salud y la 
prevención de la enfermedad a nivel comunitario. Solo se evitaran los ingresos a nivel hospitalario, si se promueve en la población en general estilos de vida más saludables.

La experiencia que llevamos a cabo en la atención a las tres personas y sus familias, demuestra la eficacia de la aplicación del proceso de atención de enfermería. El desarrollo minucioso de cada una de las etapas, combinado con una definición clara de los objetivos de la intervención de enfermería, representa una alternativa de tratamiento integral de este tipo de problemas en la comunidad. La sistematización de la práctica, nos permitió desarrollar una visión integral de nuestro trabajo, basada en una perspectiva de las necesidades individuales y familiares, bajo el enfoque del respeto a los derechos de las personas. Esto nos generó una sensación de satisfacción personal y académica, además de que nos motiva a implementar cambios en nuestro propio estilo de vida. Por otra parte nos lleva a estar más comprometidas con la profesión de enfermería, en el quehacer de ésta, al querer alcanzar un paradigma transformador para así luchar por una vida mejor.

Es necesario establecer una continuidad en la atención de las personas con quienes trabajamos, como un proceso de acompañamiento que les permita tener más herramientas para avanzar en el cuidado de su salud, esto debe establecerse a partir de una relación estrecha entre la Universidad el Centro de Salud y la comunidad.

Trabajar con población adulta con procesos mentales mórbidos es una experiencia innovadora, ya que permite abrir la mente a horizontes diferentes no solo en el área hospitalaria, sino desde la atención primaria y de esta manera se avanza hacia un concepto de salud transformador. Además trabajar con personas adultas nos ayuda a entender mayormente a dicha población y a aprender de su experiencia de vida.

\section{Bibliografía}

Adis Castro, G (1992) Desórdenes mentales en Costa Rica: observaciones epidemiológicas. San José: EDNASSSCCSS.

Caja Costarricense de Seguro Social (2000) Nuevo Modelo de Atención en Salud Mental, Gerencia médica de modernización y desarrollo, Marzo. San José: CCSS

Chávez et all (2006) Sistematización de un proceso terapéutico desarrollado con cuatro sistemas familiares, que cuentan con un miembro que presenta una alteración mental. Sistematización de Residencia Práctica presentada para optar por el título de maestría en Enfermería en Salud Mental. Universidad de Costa Rica. San José, Costa Rica.

Cohen, H. (2001). Principios de la Salud Mental Comunitaria. En Voz Pro Salud Mental. Obtenido el 6 de marzo del 2007 desde

http://portal.vozprosaludmental.org.mx

Consejo Internacional de Enfermeras (2002). Disponible en la página web: http://www.icn.ch/psmentalsp.htm

Daskal, Ana M (1992) La vida cotidiana de las mujeres. En el malestar silenciado, la otra salud mental. Santiago: Editorial Iris Internacional.

Duarte, Javier (1997) Investigación en sí (www.monografias.com/trabajos14/lainvestigacion/la-investigacion.shtml) recopilado el 06 de junio del 2006.

Escuela de Enfermería, Universidad de Costa Rica (2006). Programa del Módulo 5: Intervención de Enfermería en la Adultez en Procesos Mórbidos de la Salud. Material fotocopiado. Universidad de Costa Rica.

García, Carmen (1998). Manual para la utilización del cuestionario de salud general de Goldberg. Adaptación cubana. 
Rev Cubana Med Gen Integr 1999; 15(1):88-97 Disponible:enla página web: http://bvs.sld.cu/revistas/mgi/voll5_1_99/m gil0199.htm

Guinsberg, E (1985) Sociedad, salud y enfermedad mental. Xochimilco: Universidad Autónoma MetropolitanaXochimilco.

Jara, O. (1998) Para sistematizar experiencias: una propuesta teórica y práctica. San José: ALFORJA

Maddaleno, Matilde. (2002) Manual de medicina de la adolescencia. México D.F.: OPS

Meza, M. (2007). Atención de enfermería en los trastornos mentales según perfil epidemiológico en Costa Rica. Material del curso Procesos Mentales Mórbidos. Módulo Adultez en Procesos Mórbidos, Escuela de Enfermería: UCR.

Ministerio de Salud. (2003). Políticas Nacionales de Salud 2002-2006. Costa Rica. En: http://www.ministeriodesalud.go.cr/politicas .htm

O’Brien, P et all (2001) Enfermería Psiquiatrica. Mc Graw Hill. México, D. F: Océano.

OMS (2001) Proyecto de políticas de salud mental: paquete de orientación sobre políticas y servicios. Ginebra: OMS

OPS (2004). Situación Actual de la Salud Mental en Costa Rica. Ministerio de Salud. Costa Rica: OPS

Peplau, Hildergard (1990) Relaciones interpersonales en enfermería. Madrid: Salvat

Rodríguez, Ana L. (2004) Elisa y su incansable y dinámica Rueda de la Vida. Ensayo sobre el desarrollo del ser humano que nace $y$ crece inserto en una familia $y$ cultura determinadas. San José: Antología Modulo del Niño y la Niña Sanos(as), 2004, EEUCR. 